Voix et Images

voixetimages

\title{
Le chaos dedans et dehors
}

\section{Michel Biron}

Volume 30, numéro 2 (89), hiver 2005

Les avatars du biographique

URI : https://id.erudit.org/iderudit/011248ar

DOI : https://doi.org/10.7202/011248ar

Aller au sommaire du numéro

\section{Éditeur(s)}

Université du Québec à Montréal

\section{ISSN}

0318-9201 (imprimé)

1705-933X (numérique)

Découvrir la revue

Citer ce compte rendu

Biron, M. (2005). Compte rendu de [Le chaos dedans et dehors]. Voix et Images, 30(2), 133-139. https://doi.org/10.7202/011248ar d'utilisation que vous pouvez consulter en ligne.

https://apropos.erudit.org/fr/usagers/politique-dutilisation/ 


\section{ROM A N}

Le chaos dedans et dehors

$+++$

MICHEL BIRON

Université McGill

Quand un critique se demande ce qu'il en est de la littérature aujourd'hui, que ce soit ici ou ailleurs, c'est généralement pour regarder du côté de la colonne des pertes: absence de grands auteurs, désengagement des écrivains, triomphe de l'image sur le texte, etc. Mais n'y a-t-il pas tout de même, au travers du décentrement avéré de la littérature, quelques nouveautés? N'y a-t-il pas quelque part une colonne des gains? On peut penser, par exemple, à certains types de textes particulièrement émouvants qui n'auraient jamais vu le jour il y a quarante ans. Ce sont des textes extrêmement personnels, des récits de soi qui s'écrivent à la frontière entre la littérature et le témoignage intime. Plusieurs n'hésiteraient pas à les placer dans la colonne des pertes, comme s'ils étaient une preuve supplémentaire de ce que l'écriture soi-disant littéraire n'est plus aujourd'hui que l'expression tristement authentique d'un «vécu». Neuf fois sur dix, c'est en effet le cas. Mais il arrive que ces récits de soi soient autre chose qu'une simple thérapie par l'écriture, qu'ils ouvrent une fenêtre sur notre monde.

C'est ce qui se produit avec le récit de Pierre Monette, Premier automne ${ }^{1}$, qui évoque les dernières semaines de la vie de sa conjointe, Diane, atteinte d'un cancer des poumons et de l'œil. Pierre Monette a déjà publié des poèmes, un roman et quelques essais, il est aussi critique littéraire et professeur de littérature. Du moins, il l'était jusqu'à tout récemment, puisqu'il prend la peine de mentionner, dans la brève notice biographique qui accompagne son récit, qu'il a fait de l'enseignement «pendant trop longtemps». Cet aveu, on s'en doute, n'est pas étranger au contenu de son livre, à la perte de Diane avec qui il aura vécu durant quinze ans un amour profond, qui est en fait le véritable sujet de ce récit. La mort et l'amour, ces deux grands thèmes de la littérature universelle, sont ici ramenés à leur plus simple expression. La littérature n'est plus une matière qu'on enseigne, une série d'auteurs, de courants esthétiques, de procédés d'écriture qu'il s'agit d'expliquer à des étudiants. Elle se confond avec la vie elle-même et se présente sous la forme

$$
++
$$

1 Pierre Monette, Dernier automne, Montréal, Boréal, 2004, 211 p. 
désordonnée du journal. La beauté et l'originalité paraissent secondaires. On n'y pense guère et pourtant elles sont là, plus vraies encore de n'être pas recherchées. L'écriture oublie qu'elle est littérature et se présente comme un don, un hommage à la femme aimée en même temps qu'une méditation sur la mort: "La plupart des gens font quelque chose de leur vie; je voudrais faire quelque chose de la mort de Diane - ne serait-ce qu'un livre.» (73)

Dernier automne s'ouvre le 21 septembre, un peu avant le diagnostic définitif, et s'achève le 3 décembre de la même année, un peu après la mort de Diane. Le récit est bref, comme la maladie est fulgurante. Presque chaque jour, l'auteur enregistre les détails de la dégradation physique de sa «chum» sans jamais se cacher la réalité. Il ne livre toutefois que l'essentiel, avec une tendresse et une retenue qui empêchent le récit d'être larmoyant ou pathétique: «Diane est distraite. Elle pose les objets en mauvais équilibre, elle les renverse. Son attention vagabonde.» (13) Il devient ce qu'on appelle aujourd'hui un «aidant naturel» et il lui arrive d'exprimer sa frustration devant les soins à prodiguer et les tâches ingrates qu'il lui faut exécuter pour permettre à Diane de demeurer à domicile. Une ou deux fois, il laisse éclater sa colère, mais il n'a guère de temps pour la révolte, car les choses vont trop vite. Sa femme si pleine de vie, quelques semaines plus tôt, devient à peine reconnaissable; elle marche avec une canne, puis affirme ressembler à une poule avec le torse gonflé et les bras et les jambes ratatinés. Elle dépérit à vue d'œil, mais elle ne veut rien savoir d'un traitement qui lui permettrait, au prix d'atroces effets secondaires, de survivre quelques mois de plus. Elle n'a pas peur de mourir. Son humour, sa sérénité forcent l'admiration de ses proches, y compris des médecins qui la soignent.

Lui, en revanche, ne sait trop comment survivre à son deuil anticipé et il cache mal sa peur. Grâce à Diane, il s'était un peu réconcilié avec le monde, mais il reste, au fond, un homme solitaire aux allures misanthropes. «La compagnie de la plupart des humains n'est pas d'un grand intérêt» (36), se plaint-il. On comprend pourquoi il cite en exergue l'écrivain américain Henry David Thoreau, qui avait trouvé refuge au fond d'un bois, près du fameux étang Walden auquel il consacra son grand livre. Mais Pierre Monette ne partage pas le transcendantalisme de Thoreau et ne croit qu'à la matérialité corporelle de l'être. Au moment où Diane meurt, il écrit: «Elle n'était rien de plus que ce corps qu'elle a toujours été; elle était totalement ce qu'elle avait été.» (196) Il ne sait pas comment il fera pour se retrouver sans elle, pour n'être plus deux, mais un seul. Il parle peu de lui-même, mais c'est toute la fragilité de l'être qui s'exprime à travers ce récit de soi.

Qu'est-ce qui fait que ce récit sans ambition littéraire acquiert un tel poids de vérité? N'est-ce pas justement parce qu'on n'y sent jamais l'artifice, l'intention littéraire? N'est-ce pas aussi parce que ce récit oppose à la logorrhée actuelle, c'està-dire au bavardage (médiatique, littéraire, savant, etc.) et aux interminables discours du moi un langage fondé sur une parole qui n'a d'autre qualité que celle d'être juste, de prendre la mesure des choses à un moment où l'existence frôle dangereusement le vide. Nul désir de postérité dans le geste d'écrire, mais plutôt un réflexe de survie, comme si l'écriture était le seul moyen de ne pas perdre pied, de faire face au néant de la solitude. Celle-ci n'est pas uniquement l'objet du texte, mais devient 
le moteur même d'une écriture qui, au lieu de se replier sur l'univers intime, se tourne, presque malgré elle, vers le monde extérieur. On ne sait pas trop si l'écriture parvient à réparer la solitude, mais on comprend que la solitude nourrit l'écriture et lui redonne ce caractère de nécessité qu'elle n'a presque plus dans nos vies ordinaires. Le livre est beau d'avoir été si naturellement dicté par la réalité.

L'excès de parole, la romancière d'origine belge Paule Noyart y répond d'une toute autre manière, même si son dernier roman, Les pékinois de monsieur Chang ${ }^{2}$, a pour figure centrale, comme dans le récit de Pierre Monette, une femme victime d'un grave cancer. Aux paroles creuses ou faussement consolatrices de ceux qui vivent dans l'innocence, Paule Noyart oppose le langage blessant et un rien délirant d'Amanda. À l'inverse de Diane, toutefois, elle n'est pas très sympathique, c'est le moins qu'on puisse dire. La maladie n'arrange pas son caractère, bien au contraire. On s'en fait une idée dès la scène d'ouverture alors qu'Amanda s'occupe des funérailles d'un ami rencontré à l'hôpital, Alex, un vétéran de guerre qui a l'air d'appartenir à un autre siècle. Il laisse dans le deuil un chien, qu'Amanda va récupérer et baptiser Alex 2 en l'honneur de son maître défunt. La cérémonie funèbre est expédiée, l'ami est enterré dans le cercueil le plus économique («Une simple boîte fera l'affaire» (12), explique-t-elle à l'homme des pompes funèbres), tout cela sous l'œil narquois d'un enfant appelé Christian qui se passionne pour la mort et la décrépitude. De retour chez elle, la narratrice pleure un peu. Mais elle ajoute, pour couper court aux épanchements: "Ça a fait plaisir à tout le monde. Mais je pleurais surtout pour le chien qui allait d'une pièce à l'autre sans trouver l'odeur de son maître.» (13) Autour d'elle, personne n'échappe à son cynisme: sa sœur Laurette, sa mère, madame Éliane, qui est chargée de ses soins et qui l'accompagne chaque semaine lors de ses traitements de chimiothérapie, son mari Richard (Riri), qui attend qu'elle guérisse avant de la quitter, son médecin Marc, qui fut son ancien amant, etc. Le seul qui soit à la hauteur de son humour noir comme le charbon de ses terrils d'enfance (nous sommes en Belgique, dans la vieille Wallonie industrielle), $c^{\prime}$ est le petit Christian. Et monsieur Chang avec ses pékinois, qu'Amanda va visiter régulièrement pour obtenir les pilules de bonheur grâce auxquelles elle parvient à survivre. Il faut cela, en effet, pour passer à travers les traitements de chimiothérapie et, plus encore, pour supporter les souvenirs d'une enfance pénible et les images douloureuses du père détesté à qui elle a refusé le pardon ultime alors qu'il était sur le point de mourir.

Tout cela aurait dû donner un roman élégiaque et assez insupportable, mais l'écriture de Paule Noyart est si vive, si mordante qu'on en oublie le côté agaçant du personnage. Les phrases, portées par le plaisir des mots et des images, s'enchaînent à toute vitesse et tant pis pour la belle syntaxe: «La lucidité, c'est comme la peste.

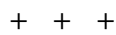

2 Paule Noyart, Les pékinois de monsieur Chang, Montréal, Leméac, 2004, 139 p. 
De gros bubons vous poussent dans la tête, ça fait mal. Puis ça éclate. Ce qui en sort, c'est des idées pas marrantes du genre pour combien de temps j'en ai et je fais quoi avec ça.» (108) Il y a chez Paule Noyart une urgence d'écrire et une passion enfantine pour le langage qui rachètent le cynisme d'Amanda. Ce qui lui permet d'écrire cette phrase magnifiquement désespérée à la toute fin, alors qu'Amanda semble provisoirement sauvée du cancer: «Tout est beau, malgré le chaos dedans et dehors.» (139)

Après le succès retentissant de Putain ${ }^{3}$, Nelly Arcan avait dit, lors d'une conférence prononcée à l'Université du Québec à Montréal, que le deuxième récit auquel elle travaillait allait être d'une encre bien différente. Il faut croire qu'elle a changé d'idée entre-temps puisque Folle ${ }^{4}$ reprend exactement là où Putain avait laissé le lecteur et dans un style assez semblable qui relève de l'autofiction. Au début de Putain, la narratrice disait bien que rien ne pourrait l'arrêter, que le récit, une fois lancé, aurait quelque chose d'inépuisable. Le voici donc qui reprend son élan avec la même intensité et qui raconte un autre désastre, non plus celui du corps mille fois traversé par les hommes, mais le désastre d'une rencontre amoureuse vouée au malheur. En surplomb, comme pour garantir que nous sommes bel et bien en face d'un destin tragique et peut-être aussi pour que ce récit de soi ne verse pas dans l'infinie répétition, la narratrice de Folle, c'est-à-dire Nelly Arcan, ajoute, se donnant sans le vouloir les allures d'un personnage romantique, qu'à quinze ans elle avait décidé d'en finir avec sa vie le jour de ses trente ans. Au moment où commence son histoire d'amour, elle a vingt-neuf ans.

Elle et lui se sont rencontrés dans un loft de Montréal, lors d'un after hour techno organisé rituellement au début de chaque saison par un regroupement de DJ, appelé Orion. Nous sommes au cœur du Montréal branché, jeune et riche. Il est Français, journaliste pigiste et il rêve d'écrire un roman parlant de sites pornos. Ils font l'amour, ou plutôt ils baisent sans arrêt, de toutes les manières possibles, mais sans jamais le moindre érotisme. Le sexe est ici une mécanique impersonnelle, une sorte de loi physique qui détermine non seulement les rapports amoureux, mais toute forme de relation humaine. "De toute façon tout le monde sait que notre époque est de communication et que la communication veut dire la chance pour tous de se branler sur le Net dans la nouveauté, dans le dernier cri du primitif et devant ce qui nous chante comme des orifices sous les aisselles ou des fillettes de dix ans qui se meurent de sucer des queues.» (31-32) On appelle cela l'esthétique «destroy».

La différence entre Putain et Folle, c'est que le premier récit parlait de la relation de la narratrice avec des hommes anonymes, qui n'avaient pas besoin

$$
++
$$

3 Nelly Arcan, Putain, Paris, Éditions du Seuil, 2001, 187 p.

4 Id., Folle, Paris, Éditions du Seuil, 2004, $205 \mathrm{p}$. 
d'exister individuellement pour donner de la consistance au récit. Il était normal que la narratrice soit le seul personnage véritable, puisque tous les autres ne faisaient que passer. Dans Folle, la relation s'établit avec un homme en particulier, mais il est vide comme le monde auquel il appartient. Ce journaliste est un pur produit de son époque; il croit dur comme fer à la nouveauté de ce qu'il fait, il est fasciné par la révolution technologique (c'est un as de l'écran, un «computer-geek» [100]), il se regarde le sexe comme Narcisse son visage. Il a d'abord connu Nelly à l'écran, chez Christiane Charrette, alors qu'elle parlait de son premier livre Putain. Le pouvoir de séduction de l'image est à la base de ses fantasmes amoureux, d'où l'espèce de malentendu qui s'installe lorsqu'il se retrouve devant la vraie Nelly. Elle et lui se sont-ils vraiment aimés? Ils y croient durant quelques mois, avec une intensité éphémère qui, elle aussi, semble l'expression parfaite d'une époque «haute vitesse». Mais derrière la folie amoureuse se cache une folie beaucoup plus grave, une folie absolue, qui n'a pas d'objet véritable en dehors d'elle-même. Une folie de l'absence au monde et à soi-même et c'est cette absence même qu'incarne, si l'on peut dire, cet homme amoureux d'images à qui Nelly adresse Folle. Mais elle parle dans le vide, ayant déjà décidé qu'elle n'enverrait pas cette longue lettre. Après la rupture, tout la renvoie à sa propre solitude, à commencer par un avortement sordide, suivi d'une période de réclusion dans son trois pièces de la rue Saint-Denis, où elle regarde les cent cinquante épisodes de X-Files. Même ensuite, lorsqu'elle sort un peu, c'est pour aller au cinéma L'Amour, accompagnée d'un ami qui ne peut pas faire grand-chose pour elle, sinon la protéger de loin contre d'éventuels violeurs. Les humains ont tous l'air d'appartenir à la catégorie des «ex». Seules deux figures semblent plus présentes et accompagnent Nelly du début à la fin, comme d'indélébiles souvenirs d'enfance: son grand-père qui lui souffle des paroles d'apocalypse et une tante experte en tarot. Ces deux personnages mystérieux arrachent Nelly à son néant quotidien et la projettent dans un temps ancien, comme si son désastre intime prenait sa source dans un désastre archaïque.

Celui-ci n'est pas sans rapport avec l'expérience de l'écriture, comme on le voit au fur et à mesure que Nelly décrit sa folie comme une course vers l'anéantissement de soi-même. Le récit de soi acquiert alors une dimension essayistique. C'est aussi par là que Folle se distingue de Putain, mais pour le mieux cette fois. Alors que la prose de Putain ressemblait à une sorte de délire verbal, celle de Folle, malgré l'usage parcimonieux des virgules, semble beaucoup plus calme, contenue et les répétitions se font nettement plus rares. Le récit est davantage écrit que ne l'était Putain. La réflexion sur l'écriture dans Folle débouche sur l'opposition suivante: d'un côté, l'écriture journalistique et efficace de l'amant, pour qui écrire est une performance quotidienne, de l'autre, l'écriture de Nelly, qualifieee par son amant de «nocive» (143). Au lieu de chercher à lui donner tort, elle s'emploie à creuser cette idée d'une écriture malsaine, maladive, nauséeuse, asociale, autodestructrice: «Chez moi écrire voulait dire ouvrir la faille, écrire était trahir, c'était écrire ce qui rate, l'histoire des cicatrices, le sort du monde quand le monde est détruit. » (168) On aurait tort de réduire une telle noirceur à une simple pose littéraire: elle vient de la vie elle-même et elle est tout aussi réelle et humaine que la folie amoureuse. 
Dans Visions volées ${ }^{5}$, son troisième roman, Rachel Leclerc invente un drôle de personnage, Frank, qui possède le don romanesque d'entrer dans la pensée d'autrui. C'est une qualité extraordinaire, bien sûr, mais c'est aussi une maladie, car Frank est sans cesse aspiré par la vie des autres. D'où la construction assez curieuse du roman, dont les cent premières pages glissent de personnages secondaires en personnages secondaires pendant que Frank, le personnage principal, se contente de jouer le rôle peu gratifiant d'agent de circulation. C'est lui le centre, mais il s'efface dès que surgit une nouvelle figure. À Montréal, où il s'est installé après avoir grandi à la campagne, nous rencontrons tour à tour sa propriétaire, Sophie, puis les autres locataires: un poète alcoolique, un professeur à la retraite et une Acadienne partie au Togo. Chacun a droit à son fragment de récit sans qu'on sache trop pourquoi ils nous sont ainsi présentés.

L'histoire de Visions volées commence toutefois à prendre forme avec l'arrivée de la mystérieuse Erika, qui habite la chambre numéro 4, celle du grenier que l'on appelle la chambre de Barbe-Bleue, car elle est peuplée de fantômes et contient les secrets de la maison. Erika a quitté Prague à la recherche de son frère Karel, jeune marchand d'art disparu depuis quelque temps sans laisser d'adresse. Avec l'aide de Frank, qui est devenu entre-temps son amant, Erika retrouve bientôt la trace de son frère, mais c'est pour découvrir qu'il a été torturé, violé, puis assassiné de façon aussi sordide que gratuite. Son cadavre attaché à un arbre et abandonné à l'hiver, quelque part au nord de Montréal, est enveloppé d'une étrange et morbide sculpture de glace. Quelques semaines plus tard, Erika retourne à Prague sans prévenir Frank. Alors s'amorce la «mue » de Frank, qui s'en va à la recherche d'Erika, dans cette Prague enchanteresse où vécut un certain Franz Kafka. Ce n'est plus le même univers, ni le même récit: du je initial, on passe au il, comme pour illustrer le changement radical du personnage. «Je me détache, tellement que bientôt je ne pourrai parler de moi qu'à la troisième personne. Ce ne sera pas de l'affectation: je deviens un autre, je me vois devenir.» (112) Cette astuce narrative rappelle un récit de Jacques Ferron, «La charrette», qui passait lui aussi tout à coup du je au il. Mais la mue du personnage fait surtout penser à La métamorphose de Kafka, où Grégoire, voyageur de commerce, se transforme en vermine tout en gardant son âme humaine.

Comme celle de Grégoire, la transformation de Frank participe étroitement de la «découverte de soi» (119). Après s'être absorbé jusque-là dans les histoires d'autrui, voici que Frank entre, ou paraît entrer, dans sa propre histoire. Il va y perdre la trace d'Erika, perdre son allure de touriste, perdre aussi ses bagages, ses papiers, son argent, bref tout ce qui pourrait servir à l'identifier; il va même y laisser son don de voleur de visions. Condamné à l'errance, il renonce à entrer dans le merveilleux château de Prague (encore Kafka...) et doit finalement admettre qu'il

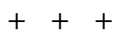

5 Rachel Leclerc, Visions volées, Montréal, Boréal, 2004, 278 p. 
ne sait plus ce qu'il est venu chercher dans cette ville. Peu à peu, il se laisse glisser dans une sorte de renoncement absolu, au point de devenir un clochard pragois, sans feu ni lieu. Frank n'est alors plus personne, il n'a pas plus d'identité qu'un mendiant ordinaire. "Avec ses loques il pourrait être de partout, ses lunettes de soleil et son gobelet de carton, de partout et enfermé dans son propre regard, dans l'humilité de ses épaules rentrées» (179). À côté de lui, sur le flanc d'une église, une femme sourde et muette dort avec son enfant dans une boîte de carton. Elle mourra peu après en lui laissant l'enfant dont il deviendra le tuteur involontaire. Il y a quelque chose de la fable merveilleuse dans le récit de cet homme égaré au cœur d'un monde qui lui est totalement étranger et qui le renvoie finalement à lui-même. On aurait d'ailleurs aimé arriver à Prague et entrer dans cette fable plus rapidement et ne pas en repartir si vite, tant cette partie du roman est riche et captivante. Le reste, c'est-à-dire l'intrigue échevelée du début comme la conclusion un peu fade, $\mathrm{n}^{\prime}$ est pas à la hauteur de ce chapitre central. C'est dommage, car le roman y perd son unité et surtout l'intensité des pages consacrées au «Château». Visions volées se lit malgré tout comme un récit tantôt réaliste tantôt énigmatique sur l'errance d'un individu et sur l'idéal de fraternité. On y retrouve l'écriture à la fois précise et poétique qui fait de Rachel Leclerc l'une des voix les plus talentueuses de la littérature québécoise contemporaine.

Les quatre romans ou récits réunis dans cette chronique appartiennent à l'évidence à des esthétiques très différentes, même si l'on retrouve dans chacun d'eux un je à la quête de lui-même. Encore une fois, comme c'est presque toujours le cas avec la littérature contemporaine, il paraît futile de chercher à tout prix une ressemblance formelle ou thématique quand c'est justement l'hétérogénéité de ces je qui les caractérise. Deux remarques toutefois permettent d'établir, pour conclure, un certain rapprochement. Tout d'abord, il est frappant de voir à quel point les quatre je sont seuls au monde, même lorsqu'ils sont entourés d'amis ou de proches. Le lien qu'ils entretiennent avec la société est extrêmement fragile. Il exclut d'ailleurs toute forme de violence ou d'agressivité. Au contraire, ces personnages se distinguent par leur résignation, leur fatalisme. Au lieu de s'opposer au monde, ce qui serait une façon d'y participer, ils penchent vers l'abnégation et l'effacement de soi. La seconde remarque concerne justement ce monde extérieur si pâle, si peu rassurant. Que ce soit la maladie, la mort, le suicide annoncé ou le meurtre crapuleux et gratuit, chacun de ces romans est placé sous le signe d'une fin abrupte. Il n'y a aucun refuge qui vaille: c'est au cœur de la vie intime que le désastre éclate soudain. Nous ne sommes pas à l'ère des grands bouleversements sociaux. Dans ces quatre fictions, il n'y a pas la moindre guerre, pas la moindre révolution, pas la moindre crise sociale. Pourtant, même si le bruit et la fureur du monde semblent lointains ou étouffés, on ne parvient jamais à oublier qu'il y a, pour reprendre l'expression de Paule Noyart, «le chaos dedans et dehors». 